\title{
Results of a pre-pilot study of potential test material for the external quality assessment of reticulocyte haemoglobin content
}

Sir,

The increasing sophistication of automated red cell analysis has led to the routine availability of new measures shown to have clinical utility in the assessment of iron status. Of these, reticulocyte haemoglobin content (RHC) has shown considerable promise [1,2], as it is at the reticulocyte stage of development that clinically important fluctuations in cellular measures of functional iron status are first seen. RHC has recently been recommended as a measure of choice in the diagnosis and management of iron deficiency in patients with chronic renal failure (3) creating a need for external assessment of laboratory performance. We report here the results of a pre-pilot study to assess the suitability of material for potential use in such a scheme with instruments available in the UK capable of providing this measure.

Four samples were produced by the UK National External Quality Assessment Scheme for Haematology (UK NEQAS(H)). Two ( $\mathrm{RH} 2$ and $\mathrm{RH} 4)$ were derived from a single donation from a healthy donor taken into citrate-phosphate-dextrose anticoagulant and supplied by National Health Service Blood and Transplant. The other two (RH1 and $\mathrm{RH} 3$ ) were obtained with informed consent from patients with iron-deficient primary polycythaemia treated by venesection and taken into acid-citrate-dextrose anticoagulant. All donations were processed according to the protocol used for the production of samples in the UK NEQAS $(\mathrm{H})$ full blood count scheme. Five aliquots of each sample, labelled 'Day 1 ' - 'Day 5' and each containing sufficient material for 10 
analyses, were issued to six volunteer laboratories together with an instruction sheet giving guidance on sample analysis. In order for analysis to take place over five consecutive working days, donations were collected six days beforehand and processed and despatched the following day in order to arrive at the volunteer laboratories before the weekend prior to the commencement of the trial. The volunteer laboratories comprised two users each of Abbott, Siemens and Sysmex instruments.

Mean (SD) RHC and reticulocyte percent values were obtained for each sample on each instrument over five consecutive days during August 2013. One Sysmex user requested additional samples and analysed them on two instruments. Bartlett's test was used to investigate differences in between-day precision and one-way analysis of variance to investigate differences in between-day mean values for samples analysed on each instrument. To allow for multiple comparisons only $p$ values $<0.001$ were considered statistically significant. Findings for RHC (pg; reported as $\mathrm{MCHr}$ with Abbott, $\mathrm{CHr}$ with Siemens and Ret-He with Sysmex instruments) are shown in the Table. With Abbott instruments statistically significant differences in between-day precision were found for five of the eight sets of data. Between-day mean values differed significantly in all eight data sets, with daily mean values differing by $\geq 2 \mathrm{pg}$ in six. There were fewer statistically significant differences with Siemens and Sysmex instruments.

Reticulocyte percent values showed acceptable between-day precision for all samples and for all instruments, with no statistically significant findings. There were a few statistically significant differences for between-day mean values with both Abbott and 
Sysmex instruments, although differences were small: with Abbott instruments the greatest difference between highest and lowest mean values over the five days was $0.28 \%$ (sample $\mathrm{RH} 4$ and Abbot 2), whilst with Sysmex instruments the comparable difference was $0.21 \%$ (sample $\mathrm{RH} 3$ and Sysmex 1 ). Daily mean values across all four samples and both makes of instrument ranged from $0.57-1.96 \%$. Similar results were obtained with Siemens instruments on samples from a single donation ( $\mathrm{RH} 2$ and $\mathrm{RH} 4$ ), whilst with the iron-deficient samples ( $\mathrm{RH} 1$ and $\mathrm{RH} 3$ ) mean values were higher and increased significantly over the trial period for both instruments, from $3.92-5.67 \%$ and 4.33-6.37\% with $\mathrm{RH} 1$ and $2.58-3.40 \%$ and $3.06-3.94 \%$ with $\mathrm{RH} 2$.

A suitable material for external quality assurance of RHC must permit the stable and precise measurement of this variable, by the detection of reticulocytes and their segregation from mature red cells. Our findings with three differing models of Sysmex instruments were satisfactory both in terms of reticulocyte percent and RHC; a statistically significant increase with time in the latter was seen with the iron-deficient samples $\mathrm{RH} 1$ and $\mathrm{RH} 3$ in five of six data sets although daily means differed by $<1 \mathrm{pg}$ in all. Siemens instruments also produced generally stable and precise RHC results with a slight decrease in values over time with most samples. Where statistically significant differences in between-day mean values occurred they appeared to be mainly a consequence of slight but non-overlapping differences between highly precise withinday estimates. These findings occurred despite significant increases in reticulocyte percent values with time in the two iron-deficient samples $\mathrm{RH} 1$ and $\mathrm{RH} 3$. With Abbott instruments, despite the finding of stable and precise reticulocyte percent values, 
there was a high degree of both statistically significant imprecision and between-day variation in means in $\mathrm{RHC}$ values.

Overall these findings indicate stabilised red cells processed in an identical manner to those used for routine UK NEQAS(H) blood count trials are suitable for use in external quality assessment of RHC with both Siemens and Sysmex instruments. Findings with Abbott instruments were less satisfactory and it would be prudent to repeat the study with other Abbott users to determine whether a genuine incompatibility exists between Abbott technology and the types of sample used in this study. It is the intention of UK NEQAS(H) to take forward the work reported here to the pilot study stage, to assess the feasibility of providing external quality assessment of reticulocyte haemoglobin content. 


\section{References}

1. Buttarello M, Pajola R, Novello E, Rebeschini M, Cantaro S, Oliosi F, Naso A, Plebani M. Diagnosis of iron deficiency in patients undergoing hemodialysis. Am J Clin Pathol2010;133:949-54.

2. Locatelli F, Aljama P, Barany P, Canaud B, Carrera F, Eckhardt KU, Horl WH, Macdougall IC, Macleod A, Wiecek A, Cameron S. European Best Practice Guidelines Working Group. Revised European best practice guidelines for the management of anaemia in patients with chronic renal failure. Nephrol Dial Transplant2004;19(Suppl.2):ii,1-47.

3. Ratcliffe LE, Thomas W, Glen J, Padhi S, Pordes BA, Wonderling D, Connell R, Stephens S, Mikhail AI, Fogarty DG, Cooper JK, Dring B, Devonald MA, Brown C, Thomas ME. Diagnosis and management of iron deficiency in CKD: a summary of the NICE guideline recommendations and their rationale. Am J Kidney Dis 2016 Jan 4 [Epub ahead of print] 
R F Hinchliffe ${ }^{1}$

\author{
A Mahon ${ }^{2}$ \\ W Thomas ${ }^{1}$ \\ C J Doré ${ }^{2}$ \\ C Briggs $^{1 *}$ \\ B De la Salle ${ }^{2}$
}

$\mathrm{KHyde}^{2}$

${ }^{*}$ Carol Briggs died on 25 $5^{\text {th }}$ February 2015.

${ }^{1}$ UK NEQAS(H) General Scientific Advisory Group; ${ }^{2}$ UK NEQAS(H), PO Box 14, Watford, WD18 OFJ, UK.

\title{
Acknowledgements
}

The authors thank Nisha Lad and Paul McTaggart of UK NEQAS(H) and the staff members of the participating laboratories for their assistance. The instrument manufacturers kindly provided reticulocyte count reagents free of charge to the participating laboratories. 
RFH designed the study and wrote the paper, AM produced and distributed samples and instruction sheets and tabulated results, WT provided iron-deficient samples and edited the manuscript, CD performed statistical analysis, CB enrolled participants, BDIS commissioned the study and edited the manuscript, $\mathrm{KH}$ authorised the study and critically reviewed the manuscript. All authors approved the final version of the paper. 
Table. Daily mean (SD) reticulocyte haemoglobin content (pg) values from Abbott Sapphire instruments, Siemens Advia 120 instruments and Sysmex instruments $\left(1\right.$, XN1000; 2, XE5000; 3, XE2100). $\neq$; no results available. ${ }^{a, b, c ;}$ data based on 8,9 and 4 replicate analyses respectively. Statistically significant differences $(p<0.001)$ in \# between-day precision and 1 between-day values respectively.

\begin{tabular}{|c|c|c|c|c|c|c|c|}
\hline Sample \& Day & Abbott $1 \mathrm{MCHr}$ & Abbott $2 \mathrm{MCHr}$ & Siemens $1 \mathrm{CHr}$ & Siemens $2 \mathrm{CHr}$ & Sysmex 1 Ret-He & Sysmex 2 Ret-He & Sysmex 3 Ret-He \\
\hline RH 1 Day 1 & $22.2(0.11) \# \Phi$ & $22.4(0.45) \# \Phi$ & $23.5(0.08)$ & $23.6(0.10)$ & $25.2(0.16)$ & $24.9(0.30)$ & $23.7(0.34) 9$ \\
\hline Day 2 & $20.9(0.44)$ & $23.0(0.90)$ & $23.3(0.07)$ & $\neq$ & $25.6(0.16)$ & $25.0(0.36)$ & $23.9(0.25)$ \\
\hline Day 3 & $22.5(0.63)$ & $25.2(1.14)$ & $23.1(0.12)$ & $23.1(0.11)$ & $25.8(0.23)$ & $25.1(0.32)$ & $24.4(0.49)$ \\
\hline Day 4 & $22.2(0.94)$ & $25.5(1.90)$ & $23.1(0.10)$ & $23.2(0.05)$ & $25.8(0.11)$ & $25.1(0.40)$ & $24.5(0.39)$ \\
\hline Day 5 & $20.4(0.28)$ & $22.6(0.41)$ & $22.9(0.08)$ & $23.0(0.10)$ & $26.1(0.29)$ & $25.5(0.30)$ & $24.6(0.39)$ \\
\hline RH2 Day 1 & $28.8(0.11) \rrbracket$ & $28.8(0.53) \# ף$ & $30.5(0.16) \rrbracket$ & $30.2(0.10) \rrbracket$ & $32.0(0.27)$ & $31.2(0.70)$ & $30.7(0.33)$ \\
\hline Day 2 & $27.0(0.20)$ & $30.1(0.87)$ & $30.4(0.25)$ & $\neq$ & $31.8(0.15)$ & $31.5(0.61)$ & $30.5(0.65)$ \\
\hline Day 3 & $29.1(0.21)$ & $31.4(0.66)$ & $30.3(0.19)$ & $29.8(0.22)$ & $31.8(0.34)$ & $30.9(0.37)$ & $30.2(0.36)$ \\
\hline Day 4 & $28.7(0.57)$ & $31.2(0.60)$ & $30.1(0.27)$ & $30.2(0.14)$ & $31.8(0.36)$ & $31.0(0.59)$ & $30.6(0.59)$ \\
\hline Day 5 & $26.7(0.47)$ & $29.4(0.27)$ & $30.1(0.22)$ & $29.8(0.17)$ & $32.0(0.35)$ & $31.0(0.89)$ & $30.3(0.52)$ \\
\hline RH3 Day 1 & $23.6(0.15) \# \Phi$ & $24.1(0.29) \Phi$ & $25.6(0.14)$ & $25.2(0.14) \uparrow$ & $27.3(0.16) \uparrow$ & $26.4(0.35) \Phi$ & $25.9(0.24)$ \\
\hline Day 2 & $22.5(0.31)$ & $25.7(1.13)$ & $25.6(0.11)$ & $\neq$ & $27.4(0.22)$ & $26.6(0.28)$ & $26.1(0.32)$ \\
\hline Day 3 & $24.0(0.20)$ & $25.7(0.89)$ & $25.7(0.11)$ & $25.5(0.13)$ & $27.6(0.24)$ & $26.7(0.28)$ & $26.1(0.29)$ \\
\hline Day 4 & $23.5(0.45)$ & $25.6(0.82)$ & $25.6(0.18)$ & $25.9(0.09)$ & $27.7(0.12)$ & $26.8(0.23)$ & $26.8(0.33)$ \\
\hline Day 5 & $22.0(0.40)$ & $24.1(0.28)$ & $25.7(0.07)$ & $25.6(0.13)$ & $28.0(0.25)$ & $27.3(0.24)$ & $26.6(0.35)$ \\
\hline RH4 Day 1 & $28.1(0.47)^{a} \# \llbracket$ & $29.2(0.28) \pi$ & $30.4(0.18) \uparrow$ & $30.1(0.77) \#$ & $31.8(0.25)$ & $31.1(0.62)$ & $30.5(0.49)$ \\
\hline Day 2 & $27.0(0.30)$ & $31.3(1.15)$ & $30.4(0.18)$ & $30.2(0.20)^{b}$ & $31.8(0.35)$ & $31.0(0.68)$ & $30.7(0.43)$ \\
\hline Day 3 & $29.0(0.30)$ & $30.5(1.15)$ & $30.2(0.20)$ & $29.7(0.13)^{c}$ & $31.7(0.41)$ & $30.9(0.48)$ & $30.5(0.52)$ \\
\hline Day 4 & $28.5(0.68)$ & $30.6(0.79)$ & $30.2(0.16)$ & $30.3(0.15)$ & $31.8(0.24)$ & $31.6(0.47)$ & $30.5(0.57)$ \\
\hline Day 5 & $26.8(0.51)$ & $29.4(0.20)$ & $30.2(0.14)$ & $29.8(0.12)$ & $31.9(0.37)$ & $31.5(0.51)$ & $30.4(0.54)$ \\
\hline
\end{tabular}

